\title{
LinK-LeVEl Performance Evaluation OF RELAY-BASED WIMAX NETWORK
}

\author{
James D. Gadze ${ }^{1}$ Griffith S. Klogo ${ }^{2}$ and Theophilus Anafo ${ }^{3}$ \\ ${ }^{1,3}$ Department of Electrical Engineering, Kwame Nkrumah University of Science and \\ Technology (KNUST), Kumasi, Ghana \\ ${ }^{2}$ Department of Computer Engineering, Kwame Nkrumah University of Science and \\ Technology (KNUST), Kumasi, Ghana
}

\begin{abstract}
Relay technology promises appreciable network throughput and coverage enhancement which is required for high speed wireless cellular systems to function to their optimum. This paper is focused on analysing the link performance of a relay-based WiMAX network under varying conditions. Comparison is made between relay and direct link communication in terms of BER (Bit Error Rate), spectral efficiency and capacity. Effect of multipath fading and user speed on performance are investigated as well. The entire study involves a hypothetical view as well as MATLAB simulations to predict the benefit of relay deployment. This is aimed at solving pertinent issues such as coverage holes and cell edge problems which are associated with traditional non-relay based cellular networks.
\end{abstract}

\section{KEYWORDS}

Bit error rate, capacity, coverage enhancement, coverage hole, network throughput, relay-based WiMAX, spectral efficiency.

\section{INTRODUCTION}

The deployment of wireless network technologies on the basis of the level of demand for higher data transmission rates cannot be over emphasized. Technologies that promise to provide higher data rates are rapidly enticing more vendors and operators. One of the most capable prospects of such arising technologies is WiMAX (Worldwide Interoperability for Microwave Access) described by the IEEE 802.16 standard [1]. WiMAX is intended for wireless metropolitan area networks (WMAN) providing broadband wireless access up to $50 \mathrm{Km}$ for fixed stations and 5-15 Km for mobile stations [1][2][3].

Many researchers do believe that WiMAX can propel the wireless data transmission concept to a greater magnitude than expected. The development of the IEEE 802.16 (WiMAX) standard is as a result of the increasing demand for higher bandwidth needed for video, voice and multimedia communication. This has impacted immensely on the manner in which people interact or communicate as well as enjoy their entertainment.

A traditional WiMAX cellular network earmarks a BS (base station) to offer services within a given radius of coverage. The core limitation of this architecture include low spectral efficiency at cell boundaries as a consequence of low SNR (signal to noise ratio) and coverage holes as a result of shadowing caused by land forms in mountainous areas and terrestrial obstacles such as high rise buildings in urbanized settings as depicted in figure 1.1. These factors impede drastically on signal transmission and deplete coverage to users in shadowed and far-fetched regions resulting in low data rates. 


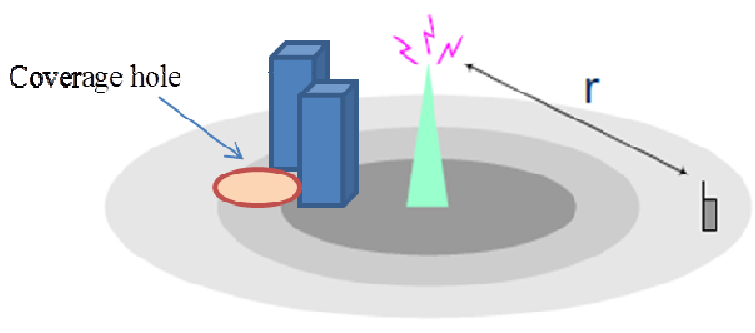

Figure 1.1: Conventional cellular coverage problems

Cellular problems of these nature have received much interests from both academic and industry researchers with some countermeasures proposed in aspects of interference management such as OFDM, MC-CDMA, etc., and also cooperation techniques such as MIMO and smart antennas. However, Spectrum efficiency near the cell edge is still poor as users suffer from enormous path loss and large inter-cell interference. Users require increased transmit power due to low bit energy to noise ratio at these regions in order to maintain bits fidelity and good throughput [3]. These prevailing issues contribute to making it almost impossible to achieve the set target of services anywhere, anytime as subscribed in the objectives of IEEE 802.16 WiMAX standard.

A simple solution to solving these problems is to strategically increase the density of BS within a geographical area which could also extend coverage to far-reached places. This approach is however cost ineffective to network operators and inefficient when few users are to be served. The use of a simple form of a BS called relay station (RS) is the rapid and cost effective way of deploying the network infrastructure [4]. Relays do not require E1 or T1 backhaul connection to communicate with BS that has link connection with some portion of their air link bandwidth [3]. User information from a nearby mobile station (MS) or user can be forwarded to a base station with the help of the relay station as shown in figure 1.2. Signal coverage can be effectively extended while enhancing overall throughput via the use of relay.

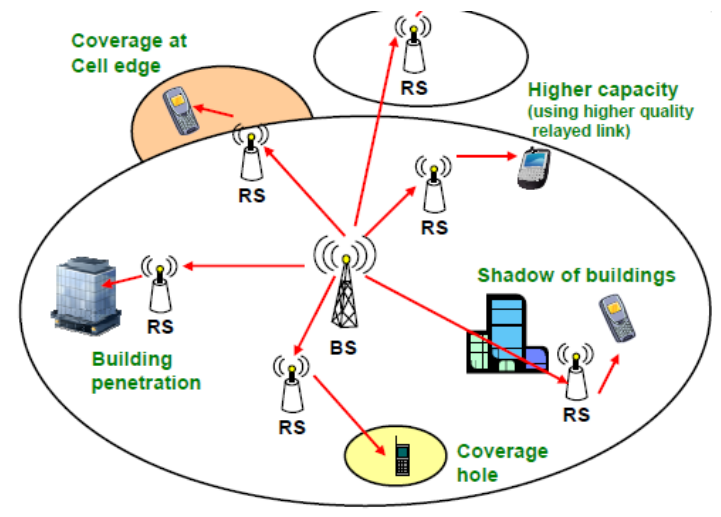

Figure 1.2: Typical Relay WiMAX Network

Types of relays that can be deployed include NT-RS (non-transparent relay station) and T-RS (transparent relay station) [5]. NT-RS are usually deployed to offer coverage at cell edge or beyond the coverage of a BS. This offer total connectivity interface between the BS and MS where traffic signal together with control signals and preamble are transmitted through relay. The other type; T-RS is deployed within BS coverage area to relay only traffic signal between BS and MS. BS send control information to MS directly in this mode. T-RS are basically used to improve throughput and capacity within the cell [6]. 
In this paper, we investigate the link-level performance of communication through relay and compare with direct link communication in a single WiMAX cell scenario in a mobile environment. Link-level performance evaluation is significant to the assessment of the behavior of a single communication link under varying condition which could provide essential results necessary to estimate the potential benefit of utilizing relays in solving the issues of coverage holes and reduced spectral efficiency at cell boundaries. A study in this direction will inform the choice and proper deployment of relays which will enhance coverage and overall cell throughput. The rest of the paper is organized as follows: Section II presents the characteristics of a relaybased network. Section III presents the some relay protocols. In section IV, link-level simulation is performed to investigate link performance calculation of BER, spectral efficiency and capacity.

Section V summarizes the results of the thesis and informs a potential direction leading to future research.

\section{Characteristics Of Relay-BaSed Networks}

Relay-based wireless network is a multi-hop design having a fixed infrastructure in which a Relay Station (RS) forms a different entity besides the BS and its associated Mobile Stations (MSs). Typically, a few low cost relays and their associated BSs form a relay-based system. Relay systems are mostly considered applicable in the network operator contexts as the operator plans and deploys the wireless access network. These settings are naturally characterised by a treebased routing setup in which end user terminals connect to BS via short two to three hops routes.

The IEEE 802.16j-2009 Relay WiMAX is a typical example of this architecture. Authors in [7][8] introduced the basic theories and principles of relay WiMAX presenting most of the major modifications in the IEEE802.16j standard. While authors in [9] and [10] have detailed the key concept and benefits of this new network design providing motivation and features relating to relay-based system design. They particularly indicate throughput enhancement, coverage enhancement and deployment cost as the three main benefits derived from relay-based architecture compared to conventional single-hop system. Each of these key benefits is further detailed below.

\subsection{Throughput Enhancement}

Relay design is expected to increase the throughput for users at the cell edge of a WiMAX system. Notably, subscribers at cell edges may be required to communicate at reduced rates [11] due to low received signal strength at these regions. In addition, relay-based design is expected to increase system capacity by deploying relays such that it enables more aggressive spatial re-use.

\subsection{Coverage Extension}

The use of a relay is probable to improve coverage in severely shadowed regions as well as extend the coverage range of a BS. In either case, coverage is improved via the RS transmitting from an advantageous location nearer to a disadvantaged user [12][13].

\subsection{Cost Reduction}

There is a potential cost gains in relay based networks deployment than single hop wireless systems. Relay stations are relatively cost effective than base stations. However, wider coverage and enhanced throughput can be achieved at low cost by adding relays in a network which is reasonable than deploying only expensive base stations in order to offer good coverage and enhanced capacity [5]. Relays which have lower complexity compared to BSs are expected with no wired connection to the backhaul [9] which is significant in providing a good cost performance in deploying a cellular network. 


\section{Relays Protocols}

Relay protocols are classified based on the signal processing techniques they employ. Authors in [14] proposed two main techniques namely, Amplify and Forward (AAF) and Decode and Forward (DAF) which are eminent to process the received signal at the relays. Each technique is described below.

\subsection{Amplify-and-Forward}

AAF protocol is mostly used in analog repeaters which simply amplifies and forwards the received signal in the downlink and uplink. This method offer an advantage in terms of delay however, certain issues arise including: increase in system noise level, increase in interference level due to lack of specific scheduling and erroneous data is forwarded without correction over multiple links [14].

\subsection{Decode-and-Forward Scheme}

In DAF scheme, received signal at the relays are fully decoded and re-encoded before signal is retransmitted which offer some advantages for the management of radio resource. Resource allocations to both relay and access links becomes quite easier making this scheme a worthy candidate for OFDMA-based systems. Additionally, this scheme is appropriate in environment where heterogeneous modulation and coding schemes are used such as in OFDMA systems.

However, this scheme define some latency issues as some amount of processing time is required for the signal decoding and re-encoding process [14].

\subsection{Related Work}

There has been a substantial work available in literature on relay protocols many of which are on analytical performance comparison of Amplify and Forward (AAF), Decode and Forward (DAF) and other relay protocols.

In [15], authors considered both AAF and DAF relay schemes investigating outdoor-to-indoor relay communication links and quantifying the end-to-end channel capacity. In measuring the links constituting the relay transmission, Real-time sounder was utilized. They established the extent to which capacity is improved when DAF relay scheme is used compared to when AAF is utilized. However, they did not establish the effect of latency and resource consumption in their model.

In [16], DAF Relay is used to allow for opportunistic relay of packets which helped to propagate redundant signals over multiple paths. The authors utilised this approach to combat the channel noise and fading effects. They investigated the use of packet combining in cooperative diversity scenario which exploits the inherent spatial diversity along with the inherent time diversity in Automatic Receive Request (ARQ) retransmissions. Additionally, their approach sought to save resource by relaying packets only at instants when direct transmission fails with performance measures in BER and throughput in AWGN and also in multipath fading.

System level performance simulation is employed in [17] with a simple AAF relay protocol operating in full duplex mode in a multihop environment. The authors investigated bandwidth efficiency and the associated service outage performance for different relay scenarios which aimed at improving carrier-to-interference ratio in heavily shadowed region. The issue with their system is the minimal throughput gain experienced by relays in open space due to co-channel 
interference from relays in neighbouring cells. Meanwhile, increased system capacity was demonstrated by fully reusing the frequency in all relays while improving the per-user data rate in cell edges and heavily shadowed regions. Authors in [18] investigated the performances for fullduplex in two-way AAF relay channel consisting of two source nodes and a single relay node in terms of outage probability and ergodic capacity. They were able to establish better performance in the presence of loop interference in full duplex mode than in two non-orthogonal half duplex channels.

A situation of power allocation at relays was addressed in [19] to increase the transmission rate in a non-orthogonal AAF relay network where amplified signals are simultaneously transmitted at the same time and with the same frequency. A unified power allocation in AAF approach is presented in [20] in multi-hop OFDM relaying systems. Authors considered a short and long-term individual and total power constraint at the BS and RSs, and formulate decentralized low complexity power allocation algorithms when links are subjected to fading. Two stages were adopted, including a power distribution phase among distinct subcarriers, and a power allocation phase among different relays.

\section{Challenges In Planning Wimax Cellular Relay-Based NETWORKS}

Network planning is quite a challenging task in rolling out a cellular network. These challenges come in many aspects and the incorporation of relays into the network gives rise to new issues. To ensure that the installed network is enhanced, it is necessary to investigate and determine certain facets in relation to the number of relays to deploy along with their location in order to address the relay selection process while mitigating the interference through frequency re-use.

\subsection{Relay Placement and Transmission}

Relay stations (RS) are the nodes found between the BSs and MSs thereby reducing the transmission distance. System performance is sensitive with respect to the number of deployed relays as well as their location. If well placed could enhance system capacity, system reliability as well as throughput per user. The problem related to relay placement has been studied in different wireless multi-hop networks such as in WLANs [21] and sensor networks [22]. The authors in [23] suggested an approach to maximise the overall system capacity by defining an algorithm for relay placement and also determining the number of relays to deploy. They clearly demonstrated significant gains via careful relay deployment. However, their work also demonstrated that no additional gain is achieved above a certain number of relays deployed. Therefore, defining the relay position under certain realistic assumptions is presented and perceived as NP-hard problem and is hence a challenging problem to solve [24]. However, if the MS distribution is assumed to be uniform in an ideal scenario according to [5], system optimization can be achieved by adopting an iterative algorithm in the placement of RS. In practice, non-uniform distributed traffic demand must be considered especially in large geographic areas. In the first stage of network deployment, the BSs locations are first determined followed by the design of the RS location algorithm to locate the RSs [4].

The IEEE $802.16 \mathrm{j}$ mobile multi-hop relay network utilizes several transmission schemes for its relays as surveyed by authors in [4][25] as captured in the standard [5]. Authors is [26][27][28][29] present some other studies on relay placement which focus on finding the best location for RS in order to improve performance by extending the coverage of the WiMAX system. In [26], the authors established a clear relationship between relay bandwidth reservation and the placement of the relay station. They also recommended an optimisation scheme where relay bandwidth reservation and relay placement are combined. 
International Journal of Wireless \& Mobile Networks (IJWMN) Vol. 8, No. 3, June 2016

\subsection{Frequency Re-use}

Radio frequency is a limited, scarce and expensive resource. It is largely improbable for same frequency to be used in adjacent cells. Frequency re-use is a mechanism that promises efficient utilisation of limited radio resource [30]. This technique has attracted the interest of several researchers in the industry. Authors in [31] gave comprehensive overview of the basic mechanism and algorithms involved in frequency planning and re-use processes for single-hop network. In [32], different re-use factors for cellular multi-hop network scenario are investigated and their respective impact on the system capacity. They demonstrated that via the use of directional antennas to limit the interference on the relay links, relay-based systems can offer significant gains over conventional single-hop systems.

\subsection{Routing Management}

Routing procedure is a significant aspect of Relay WiMAX which impact immensely on throughput. The path selection is very critical as there is a cost associated with it in terms of throughput and delay which warrant for an intelligent routing algorithm to ensure good performance of the system.

However, several routing algorithms are found in literature with different measures used to analyse the performances of different paths. Authors in [33] introduce a metric which constitutes the bandwidth unit needed to transmit a fixed amount of data by using a specific Modulation and coding scheme. The efficiency of the routed path used to transmit a bit over a medium by their metric does not account for the traffic load at the relay. Authors in [34] presented a routing scheme in multi-hop Relay-based WiMAX. Their proposed path selection maximizes the throughput of the network while minimizing latency. In finding the best route, the authors considered the Signal to Noise Ratio, the number of hops and the available link bandwidth in their decision. The achievable throughput is considered by authors in [33] and [35] by reckoning the bandwidth at the relay in terms of available free slots. This quite profound in managing the traffic load in QoS oriented wireless system. However, how to determine the available slots at each relay is not discussed. Additionally, defining the amount of resources available at the relay is directly related to the scheduling algorithm and the way the resources are managed; either centralised or distributed mode. Further in their bandwidth-based metric scheme, they did not investigate the impact of the additional signalling overhead associated with their approach. Authors in [36] conducted a system level performance in transparent relay mode demonstrating the fact that, there can only be up to $10 \%$ increase in maximum throughput in the downlink. They also argued in the article that throughput enhancement can only benefit a half of the BS coverage. Routing procedures in Relay WiMAX systems differ depending on the manner resources are managed.

Relay WiMAX routing issues seem less complex than in other wireless systems such as wireless mesh networks. However, there exist some pertinent issues in relation to path selection specifically with non-transparent relay systems.

\subsection{Resource Allocation}

Relay WiMAX significantly has an increases signalling overhead compared to the conventional single-hop WiMAX system. Therefore, to meet the different QoS requirements of each user require the design of novel scheduling algorithms as well as carefully managed signalling for an efficient resource allocation.

Authors in [37] and [38] analysed the MAC efficiency issues with Relay WiMAX proposing different schemes for the MAC efficiency optimization. A new downlink resource allocation algorithm is proposed in [37] which dynamically allocate resources on the downlink for relay 
transmission with respect to bandwidth requirement. However, this work did not consider OFDMA and scheduling not covered at all. Authors in [38] sought to reduce system overhead while improving the efficiency of utilisation of the MAC frame by proposing an aggregation scheme. The need for connection and packet aggregation which combat significant overhead was highlighted in this work. But notably, the Relay WiMAX standard different schemes from those described in [38] are defined to aggregate the relay link traffic.

Generally, most resource allocation work evolving through the years focus on the MAC efficiency optimization and does not follow the Relay WiMAX specification. These works significantly rely on system-level performance.

One aspect which is imperative with multiple relays deployment is the possibility to enable concurrent transmission at the relays which is significant for efficient utilisation of available radio resources provided interference is well managed. Resource scheduling in an urban setting under severe shadowing was investigated by the authors in [38]. They assumed in their system 4 directional antennas on both the base station and the relay station which resulted in their simulation highlighting on the advantages of directional antennas over omni-directional antennas in an urban settings. Notably, system throughput can be increased by 6 or 12 times with the proposed method compared to an omni-directional system. Additionally, this work greatly utilised spatial re-use for throughput enhancement which is further spot on via simulation studies in [40][41]. The authors emphasised the need for distributed scheduling (i.e. scheduling at the BS as well as RSs) which maximises concurrent transmissions while preventing severe interference due to wrong re-use decisions. However, the frame structure considered in these works does not conform to Relay WiMAX specifications and the relay type considered is not clear.

In [42], authors discussed several publications focusing on the management of radio resource in OFDM relay based systems which can be adapted by Relay WiMAX systems. Various approaches for dynamic resource allocation found in literature were presented.

Several of these proposals on system performance enhancement exhibit some short fall either by their signalling requirements or their complexity in implementation. Distributed scheduling is one solution discussed by authors in [42] which promises a reduced overhead and system complexity.

Largely, this review demonstrates that there is still much work needed in the performance enhancement process of OFDM relay systems and hence relay WiMAX systems. Relay WiMAX systems requires a scheduling algorithm design which takes into account the detailed issues with regards to the frame structure in transparent or non-transparent mode, signalling and QoS.

Although some clues are offered in these works with respect to achievable throughput gain of relay WiMAX systems, a lot more work is still left aloof and worth considering in terms of the best design practises and performance measures in different scenarios.

\subsection{Summary}

The Relay WiMAX is relatively a new technology with a new architecture which presents several complexities amidst an already thought-provoking wireless environment in a mobile world. Largely, system design becomes difficult due the extensive aggregate of choice available to engineers tied with a general deficiency of understanding of the different design decisions and their impact. 
The performance enhancement offered by Relay WiMAX systems over single hop WiMAX systems is influenced by numerous factors including network topology, relay mode, transmit power level at the relays and the type of antennas used. A profound understanding of the relationship between the enhancement of relay based WiMAX systems and the multiple parameters that are necessary to the system design can offer a roadmap for the deployment of relay-based WiMAX systems. The focus of the reviewed work provided is to give a better understanding of the impact of different design options on performance.

Several issues pertaining to Relay WiMAX remain unanswered as several research works are based on inaccurate assumptions. Hence, the need for much work to be done considering different system configurations which is essential in determining the most practicable scenarios of this technology. It is imperative to note that, transparent and non-transparent systems produce different issues and different approaches are required to solve them. While the reviewed literature in this section are essential and complement this paper naturally, some significant system configuration components that impact on performance are not considered.

\section{SYSTEM MODEL}

Several assumptions were made due to system complexity. We considered two hops with two kinds of users, a direct link user or MS (mobile station), communicating directly with the BS and relay link MS, communicating through relay stations to the BS as depicted in fig. 3. This is due to the fact that more than two-hop relaying without extending coverage reduces the efficiency of using RSs.

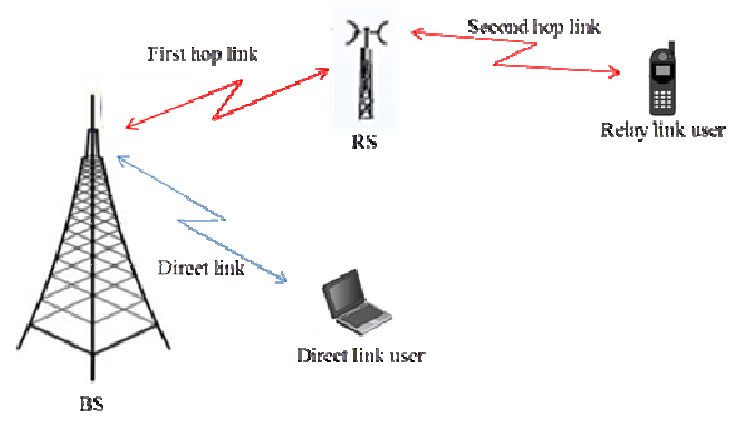

Figure 5.1: System model

The focus is on only the downlink communication whiles considering transparent relay with AAF (Amplify and Forward) relay protocol. Every node has a single omni-directional antenna and operates in half-duplex mode; hence, no terminal can receive and transmit data simultaneously.

For multipath fading, Rician fading and Rayleigh fading were considered for BS-RS link and access link respectively. For channel propagation models, SUI channel model and Cost 231 Hata model were used for BS-RS link and access link respectively.

The BS is responsible for allocating resources and is connected to the backhaul network, while the RS has no backhaul link but is wirelessly connected to the BS. The main responsibility of the RS is to relay data between the BS and MS. The one-hop links; BS to RS and RS to RS are referred to as relay links, and users served through these links are classified as relay link users.

Similarly, the links; BS to MS are referred to as direct links and users served directly by the BS are considered direct link users. 


\subsection{Channel}

In order to model such wireless channels, we can suppose that for each point in the 3-dimensional space, the wireless channel is a linear time-varying filter with following impulse response [49]

$h(t, \tau)=\sum_{k=0}^{N-1} c_{k}(t) \delta\left(\tau-\tau_{k}(t)\right) e^{j \theta_{k}(t)}$

where $t$ and $\tau$ are the observation time and application time of the impulse, respectively.

$N$ is the number of multipath components, $c_{k}(t), \tau_{k}(t), \theta_{k}(t)$ are the random time - varying amplitude, arrival-time, and phase sequence, respectively, and $\delta$ is the delta function. The output of the wireless channel for a given transmitted signal $\mathrm{s}(t)$ can be calculated by:

$$
y(t)=\int_{-\infty}^{\infty} s(t) h(t, \tau) d \tau+n(t)
$$

where $n(t)$ is the background noise.

\subsection{Multipath Fading Channels}

We assume well known fading channels such as the Rayleigh fading for the access links (i.e. BSMS and RS-MS links) and Rician fading channel for the relay link (i.e. BS-RS link). The Rayleigh fading channel is most applicable when there is no propagation along the line of sight between the transmitter and receiver, while the Rician fading channel is more appropriate when there is a dominant line of sight component at the receiver.

For BS-MS and RS-MS access links, we can precisely model via a Rayleigh distribution the amplitude distribution of the received signal as:

$$
p(\rho)=\frac{\rho}{\sigma^{2}} \exp \left(-\frac{\rho^{2}}{2 \sigma^{2}}\right), \rho \geq 0
$$

Where $\rho$ represent the received signal amplitude and $\sigma^{2}$ represent the local mean power [50]. Now according to the amplitude of the received signal probability density function (pdf) in (3), we can derive the received signal power pdf, $\gamma$, which has the exponential pdf:

$$
p(\gamma)=\frac{1}{\gamma^{*}} \exp \left(-\frac{\gamma}{\gamma^{*}}\right), \gamma \geq 0
$$

where $\gamma^{*}$ represent the received signal mean power. Similarly, for the relay (BS-RS) link, we can via Rician distribution model the amplitude distribution of the received signal as:

$$
p(\rho)=\frac{\rho}{\sigma^{2}} \exp \left(-\frac{\rho^{2}+v^{2}}{2 \sigma^{2}}\right) \mathrm{I}_{0}\left(\frac{\rho v}{\sigma^{2}}\right), \rho \geq 0, v \geq 0
$$

If $I_{n}($.$) represent a modified n^{\text {th }}$-order Bessel function of the first kind, $\frac{1}{2} v^{2}$ characterise the power of the LOS component while the power of all other scattered components denoted as $\sigma^{2}$; 
we can deduce the total mean power of the received signal $\gamma^{*}$ as:

$\gamma^{*}=\frac{1}{2} v^{2}+\sigma^{2}$

The Rician K-factor is given as the ratio between the signal power in the dominant component and the local mean scattered power [51], where $K=\frac{v^{2}}{2 \sigma^{2}}$. Rayleigh when the $K$-factor goes to zero; the pdf of $\gamma$, (received signal power) from equation (5), can be obtained by transforming random variable $\rho$ into $\gamma$ via the amplitude and power relation of the signal $\gamma=\frac{1}{2} \rho^{2}$. According to [69] the pdf of received signal power is given by:

$$
p(\gamma)=\frac{(1+K) e^{-K}}{\gamma^{*}} \exp \left(-\frac{(1+K) \gamma}{\gamma^{*}}\right) I_{0}\left(\sqrt{\frac{4 K(1+K) \gamma}{\gamma^{*}}}\right), K \geq 0, \gamma \geq 0
$$

Where $\gamma^{*}$ is the received signal mean power.

\subsection{Relay Strategy}

An MS located within BS coverage can either be served directly by BS or via RS. More often than not, the MSs will prefer an RS route only if the achievable relay link data rate (BS-RS-SS) is superior to the direct link data rate (BS-SS). The relay link data rate is influenced by the link capacities and time durations of the linking two hops. The assumption made in our case is that every node has a single antenna and operates in half duplex mode and also, data arriving equals data leaving at the RS node.

For each transmission time interval according to [5], the BS allocates sub-channels to both relay and direct links. Within a segment, if the $k_{t h}$ sub-channel represented by $a_{k, m}$ is allocated to a direct link user $m$ then;

$a_{k, m}=\left\{\begin{array}{lr}1, \text { subchannel } \mathrm{k} \text { allocated } \\ o, & \text { otherwise }\end{array}\right.$

Likewise

$b_{k, r, m}=\left\{\begin{array}{rr}1, & \text { subchannel } \mathrm{k} \text { allocated to user using } \mathrm{RSr} \\ 0, & \text { otherwise }\end{array}\right.$

Where $b_{k, r, m}$ represent $k_{t h}$ subchannel allocated to user $m$ using $\mathrm{RSr}$ (relay station r) using the second hop link [52].

Estimating the capacity of direct link in the first time slot using Shannon's formula is given by:

$$
C_{k, m}=B_{k} \log _{2}\left(1+p_{k, m} g_{k, m} / \sigma^{2}\right)
$$

With $B_{k}$ representing the bandwidth of the each channel, $P_{k, m}$ represent the transmit power on the $k_{t h}$ subchannel of BS to MS m, $g_{k, m}$ represent the gain of subchannel and $\sigma^{2}$ represent the AWGN power level [53]. 
Similarly capacity of the relay link is

$C_{k, \mathrm{r}}=B_{k} \log _{2}\left(1+p_{k, \mathrm{r}} g_{k, r} / \sigma^{2}\right)$

Where $P_{k, r}$ denotes the transmit power on the ${ }^{k_{t h}}$ subchannel of BS to RS r.

for $m$ number of users on the relay link, the above equation becomes:

$$
C_{k, \mathrm{r}, \mathrm{m}}=B_{k} \log _{2}\left(1+p_{k, \mathrm{r}, \mathrm{m}} g_{k, r, m} / \sigma^{2}\right)
$$

On the direct link, the realistic data rate in the first time slot according to [53] is given by:

$$
C_{m}=\sum_{k=1}^{K} C_{k, m} a_{k, m}, \quad m \in \mu_{D}
$$

Where $\mu_{D}$ represent a set of direct link users.

And on the relay link, in the second time subslot, data rate for user $m$ is given by:

$$
C_{m}^{(2)}=\sum_{k=1}^{K} C_{k, r, m} b_{k, r, m}, \quad m \in \mu_{R}
$$

In the first time slot, all relay link users share the data rate of RS r, therefore producing a data rate in the first time subslot for each user in the relay link as:

If $C_{r}^{(1)} C_{m}^{(1)}=C_{r}^{(1)} \cdot C_{m}^{(2)} / \sum_{m \in \mu_{R r}} C_{m}^{(2)}$

denotes data rate of RS $\mathrm{r}$ of the first hop link given by:

$$
C_{r}^{(1)}=\sum_{k=1}^{K} C_{k, r} a_{k, r}
$$

If a single user is considered as in our scenario, then $C_{m}^{1}=C_{r}^{1}$.

Now, given that $t_{B S-R S}$ represent the first time subslot and $t_{R S-M S}$ represent the second time subslot; the total duration of the downlink subframe transmission should be $t_{B S-R S}+t_{R S-M S}$. The amount of data transfer from BS to RS is equal to that from RS to MS.

$$
C_{m}^{1} \cdot t_{B S-R S}=C_{m}^{2} \cdot t_{R S-M S}
$$

To determine the average data rate of user on the relay link, we divide the amount of data received by the time required to receive it [52]. 


$$
\bar{C}_{m}=\frac{C_{m}^{1} \cdot t_{B S-R S}}{t_{B S-R S}+t_{R S-M S}}
$$

Since RS cannot receive from BS while transmitting to MS. Subsequently, using the relation in [53], we can rewrite the relay data rate of the MS as:

$$
\frac{1}{\bar{C}_{m}}=\frac{1}{C_{m}^{1}}+\frac{1}{C_{m}^{2}}
$$

\section{Simulation ReSUlts AND Discussion}

In the simulation setup, a WiMAX cell scenario of BS is considered and RS deployed within its coverage. A downlink transmission is considered with a channel bandwidth of $5 \mathrm{MHz}$ and a $2.5 \mathrm{GHz}$ carrier frequency. The BS is centralised and the RS kept in amplify and forward (AAF) mode.

First, we estimate the link condition in terms of BER between the BS, the RS and the MS as a function of bit energy to noise ratio $\left(\mathrm{E}_{\mathrm{b}} / \mathrm{N}_{0}\right)$ and subsequently spectral efficiency and the link capacities are also evaluated. We used the SUI channel model for link between BS-RS and Cost 231 channel model between BS-MS and RS-MS links while specifying the velocity of MS within the Doppler frequency. Initialization parameters and input data form the core of the program. At the time of initialization, parameters that can be set include the number of subcarriers, the nominal bandwidth, $\mathrm{CP}$ length, symbol modulation level and coding rate, range of the bit energy to noise ratio $\left(\mathrm{E}_{\mathrm{b}} / \mathrm{N}_{\mathrm{o}}\right)$ values (from 0 to $40 \mathrm{~dB}$ ) and channel model parameters for simulation. The input data stream is randomly generated. Output variables are available in MATLAB workspace while bit error rate (BER) values for different $E_{b} / N_{o}$ are stored in text files which facilitate in the plots. In our model, the simulation of the system is repeated and the number of transmitted bits and bit errors are calculated for each simulation. At the end, BER is estimated as the ratio of the total number of observed errors and the total number of transmitted bits.

The performance of the system model is evaluated using varied modulation and coding schemes such as BPSK 1/2, QPSK 1/2, QPSK 3/4, 16-QAM 1/2, 16-QAM 3/4, 64-QAM 2/3 and 64-QAM 3/4 while considering several MS velocities.

Our simulations can be summarised into 3 stages:

1. BER and Spectral efficiencies at fixed speed of MS in pure AWGN channel.

2. BER at varied speed of MS in fading channel (i.e. Rayleigh and Rician) compared to that of non-fading pure AWGN channel.

3. Capacities of the two links under study (i.e. relay and direct link).

The next sections present a set of plots to identify trends in signal quality as we change different parameters. These plots include BER vrs. $E_{b} / N_{0}$, spectral efficiency vrs $E_{b} / N_{0}$ and capacity vrs $\mathrm{E}_{\mathrm{b}} / \mathrm{N}_{0}$ plots.

\subsection{Performance measure of Relay and Direct links in AWGN channel}

In this section, we have presented various BER vrs $\mathrm{E}_{\mathrm{b}} / \mathrm{N}_{0}$ and spectral efficiency vrs $\mathrm{E}_{\mathrm{b}} / \mathrm{N}_{0}$ plots for all the mandatory modulation and coding schemes as specified in the IEEE $802.16 \mathrm{j}$ standard for both relay and direct link. Figures 6.1 - 6.14 show these plots exhausting BPSK, QPSK, 16QAM and 64-QAM in pure AWGN using OFDM comparing performances of the two links. For each cited modulation, the BER of relay and direct links are compared as well as their respective spectral efficiencies estimated. 


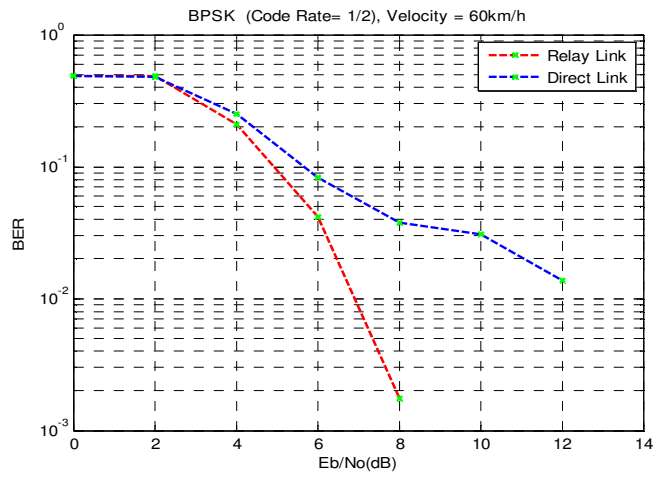

Figure 6.1: BER against $\mathrm{E}_{\mathrm{b}} / \mathrm{N}_{0}$ for $\mathrm{MS}$ at $60 \mathrm{~km} / \mathrm{h}$ via AWGN channel (BPSK-1/2)

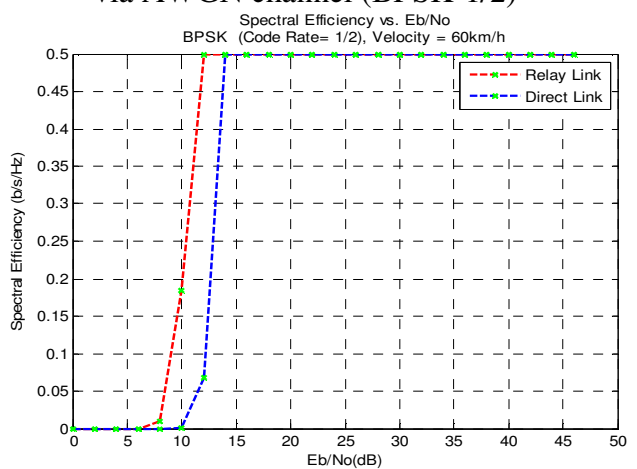

Figure 6.2: Spectral Efficiency against $\mathrm{E}_{\mathrm{b}} / \mathrm{N}_{0}$ for MS at $60 \mathrm{~km} / \mathrm{h}$ via AWGN (BPSK-1/2)

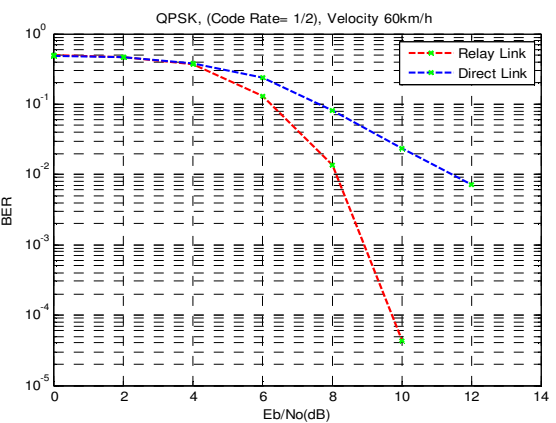

Figure 6.3: BER against $\mathrm{E}_{\mathrm{b}} / \mathrm{N}_{0}$ for $\mathrm{MS}$ at $60 \mathrm{~km} / \mathrm{h}$ via AWGN channel (QPSK-1/2)

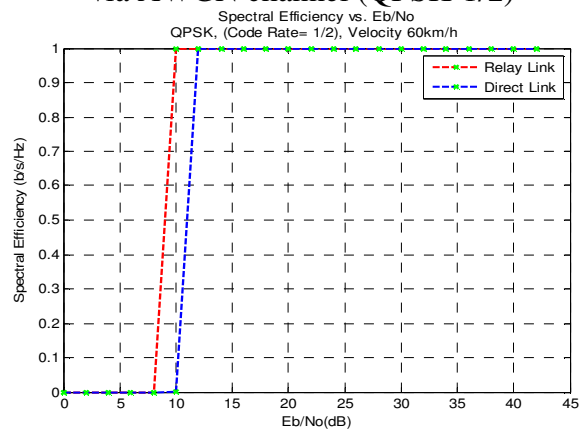

Figure 6.4: Spectral Efficiency against $\mathrm{E}_{\mathrm{b}} / \mathrm{N}_{0}$ for MS at $60 \mathrm{~km} / \mathrm{h}$ via AWGN (QPSK-1/2)

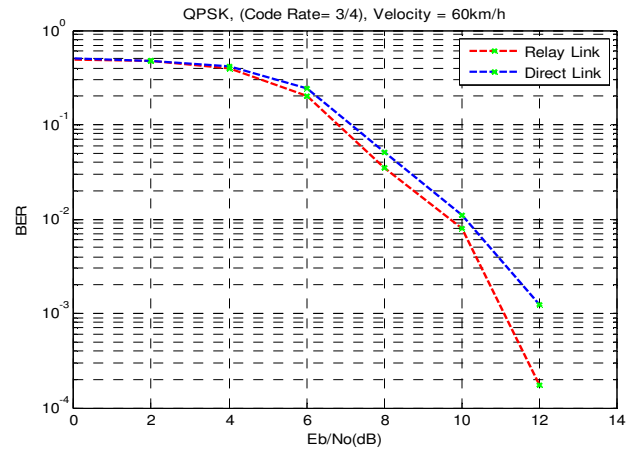

Figure 6.5: BER against $\mathrm{E}_{\mathrm{b}} / \mathrm{N}_{0}$ for $\mathrm{MS}$ at $60 \mathrm{~km} / \mathrm{h}$ via AWGN channel (QPSK-3/4) Spectral Efficiency vs. Eb/No
QPSK, (Code Rate $=3 / 4)$, Velocity $=60 \mathrm{~km} / \mathrm{h}$

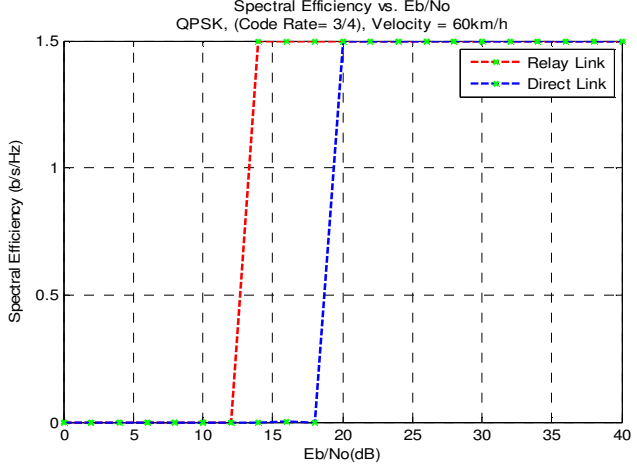

Figure 6.6: Spectral Efficiency against $\mathrm{E}_{\mathrm{b}} / \mathrm{N}_{0}$ for MS at $60 \mathrm{~km} / \mathrm{h}$ via AWGN (QPSK-3/4)

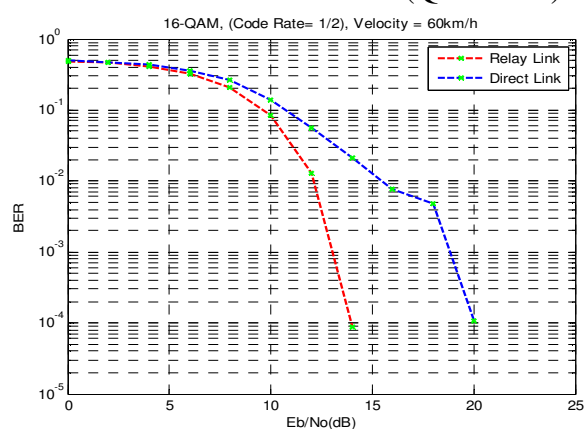

Figure 6.7: BER against $\mathrm{E}_{\mathrm{b}} / \mathrm{N}_{0}$ for $\mathrm{MS}$ at $60 \mathrm{~km} / \mathrm{h}$ via AWGN channel (16QAM-1/2)

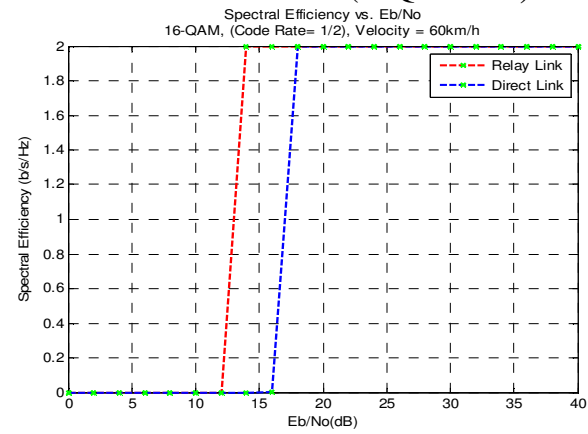

Figure 6.8: Spectral Efficiency against $\mathrm{E}_{\mathrm{b}} / \mathrm{N}_{0}$ for $\mathrm{MS}$ at $60 \mathrm{~km} / \mathrm{h}$ via AWGN (16-QAM-1/2) 


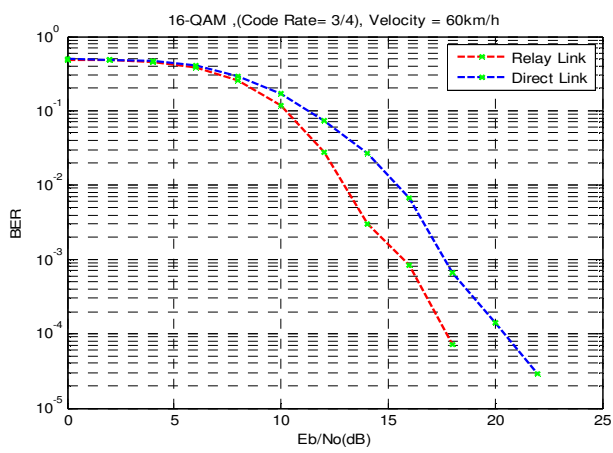

Figure 6.9: BER against $\mathrm{E}_{\mathrm{b}} / \mathrm{N}_{0}$ for $\mathrm{MS}$ at $60 \mathrm{~km} / \mathrm{h}$ via AWGN channel (16QAM-3/4)

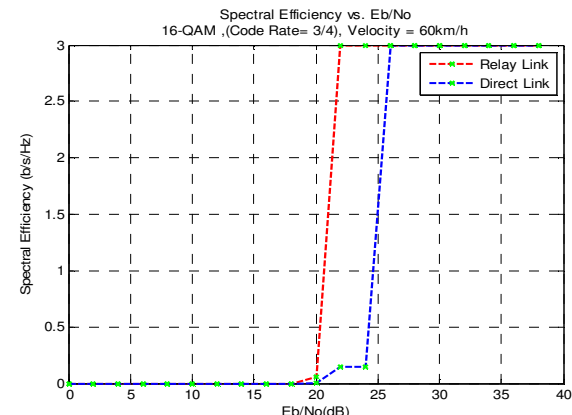

Figure 6.10: Spectral Efficiency against Eb/N0 for MS at $60 \mathrm{~km} / \mathrm{h}$ via AWGN (16-QAM-3/4)

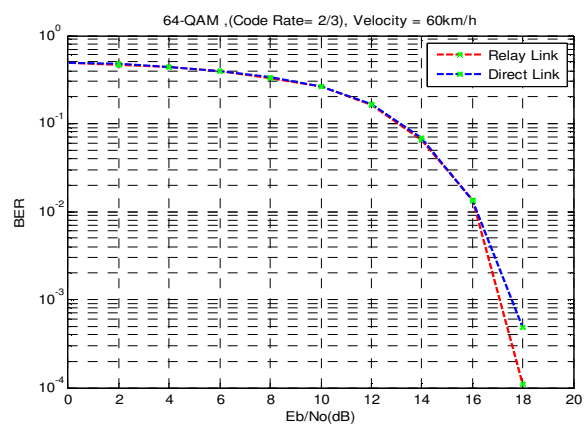

Figure 6.11: BER against $\mathrm{E}_{\mathrm{b}} / \mathrm{N}_{0}$ for $\mathrm{MS}$ at $60 \mathrm{~km} / \mathrm{h}$ via AWGN channel (64QAM-2/3)

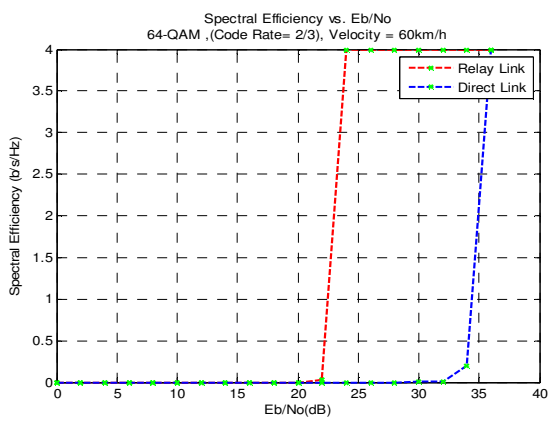

Figure 6.12: Spectral Efficiency against $\mathrm{E}_{\mathrm{b}} / \mathrm{N}_{0}$ for $\mathrm{MS}$ at $60 \mathrm{~km} / \mathrm{h}$ via AWGN (64-QAM-2/3)

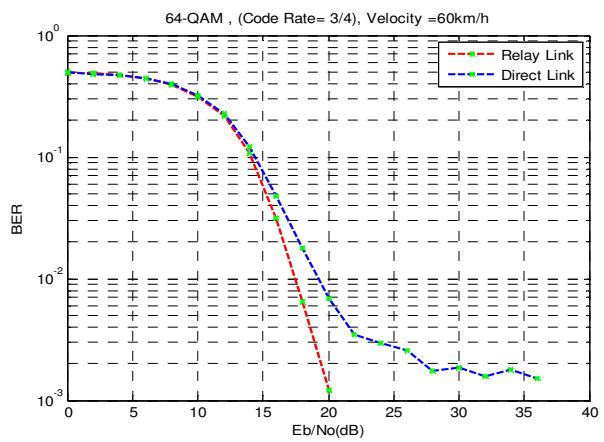

Figure 6.13: BER against $\mathrm{E}_{\mathrm{b}} / \mathrm{N}_{0}$ for $\mathrm{MS}$ at $60 \mathrm{~km} / \mathrm{h}$ via AWGN channel (64-QAM-3/4)

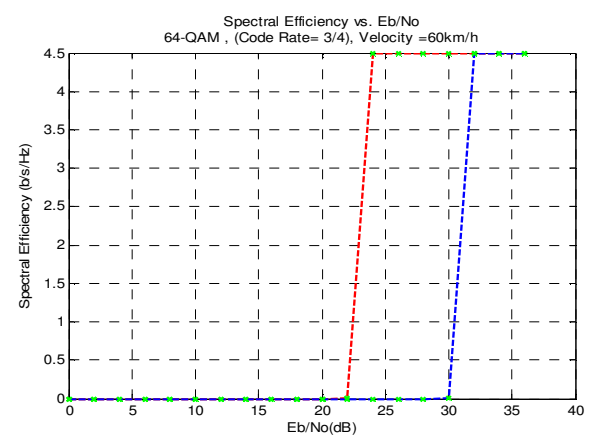

Figure 6.14: Spectral Efficiency against $\mathrm{E}_{\mathrm{b}} / \mathrm{N}_{0}$ for MS at $60 \mathrm{~km} / \mathrm{h}$ via AWGN (64-QAM-3/4

We observed that, the relay link marginally performed better than the direct link at an MS velocity of $60 \mathrm{~km} / \mathrm{h}$. It was also prominent that BPSK is more power efficient and require less bandwidth than all the other higher modulation techniques used. In case of bandwidth utilization, the 64-QAM modulation requires higher bandwidth but gives excellent data rates as compared to the lower modulations while QPSK and 16-QAM performances fall intermediate between the two extremes and need higher bandwidth and are less power efficient than BPSK. But they required lesser bandwidth and lower data rates than 64-QAM. BPSK has the lowest BER while the 64QAM has the highest BER. 


\subsection{Effect of speed and fading on Performance}

The results presented here are for simulations performed for the MS velocities of 30, 60 and 90 $\mathrm{km} / \mathrm{h}$ in fading and non-fading (pure AWGN) channels. The prime purpose of this section is in two folds. The first is to investigate the effect of speed to system performance and the next is to probe into the influence of fading channel to performance. Figures $6.15,6.16$ and 6.17 present the BER curves of QPSK-1/2, 16-QAM-1/2 and 64-QAM-2/3 with varied MS speed of 30, 60 and $90 \mathrm{~km} / \mathrm{h}$ while considering a relay link in pure AWGN channel. Figures $6.18,6.19$ and 6.20 present BER curves with same parameters in multipath fading channel.

It is clearly indicated from the results obtained that fading channels offer degraded performance compared to non-fading channels. On the side of testing, speed has a great impact on performance. It was also well noted that better performance is experienced when MS is moving at slower speed than when moving faster.

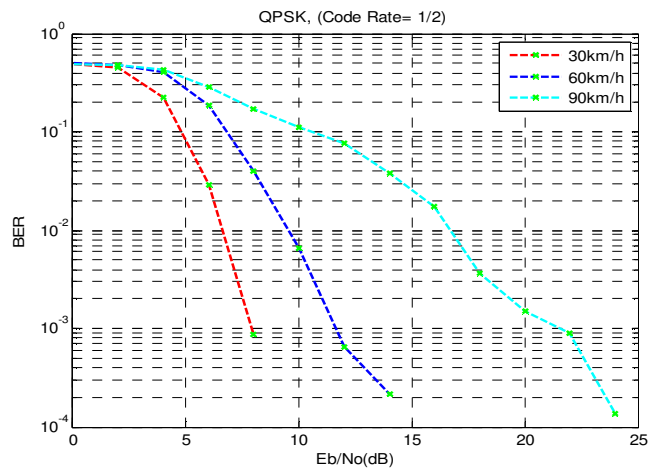

Figure 6.15: BER against $\mathrm{E}_{\mathrm{b}} / \mathrm{N}_{0}$ for $\mathrm{QPSK}-1 / 2$ in AWGN, V=30,60 and $90 \mathrm{~km} / \mathrm{h}$

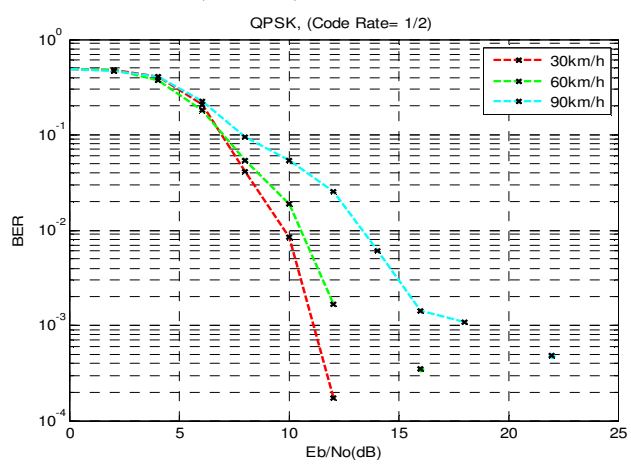

Figure 6.16: BER against $\mathrm{E}_{\mathrm{b}} / \mathrm{N}_{0}$ for QPSK-1/2 in Fading channel, V=30,60 and $90 \mathrm{~km} / \mathrm{h}$

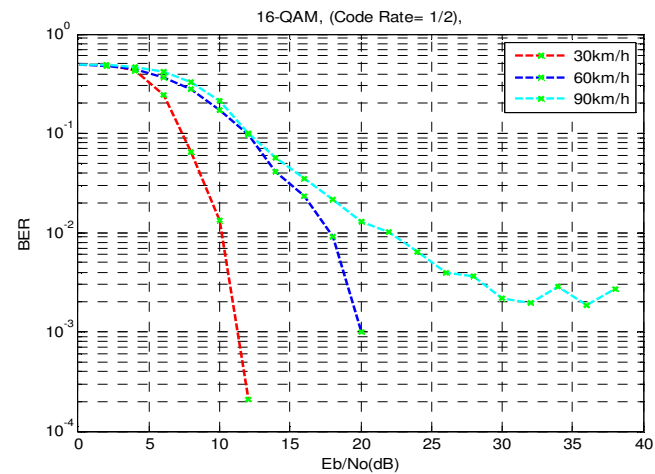

Figure 6.17: BER against $\mathrm{E}_{\mathrm{b}} / \mathrm{N}_{0}$ for 16-QAM-1/2 in AWGN, V=30,60 and $90 \mathrm{~km} / \mathrm{h}$

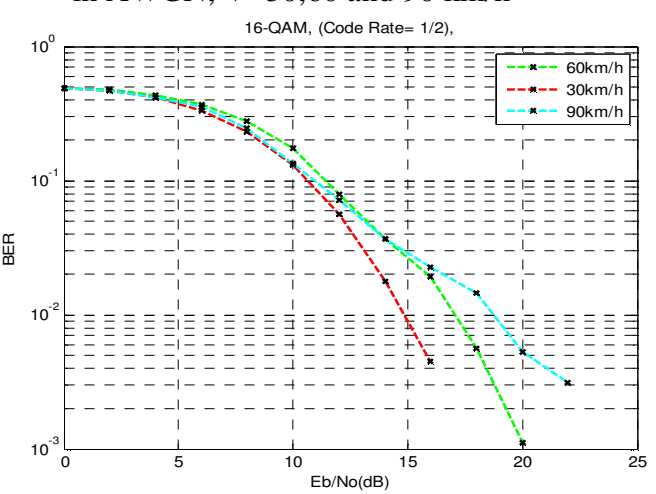

Figure 6.18: BER against $\mathrm{E}_{\mathrm{b}} / \mathrm{N}_{0}$ for $16-\mathrm{QAM}$ $1 / 2$ in Fading channel, V=30,60 and $90 \mathrm{~km} / \mathrm{h}$ 


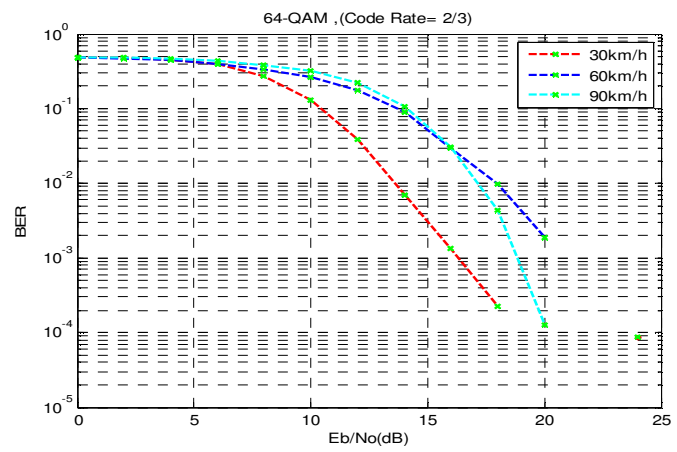

Figure 6.19: BER against $\mathrm{E}_{\mathrm{b}} / \mathrm{N}_{0}$ for 64-QAM-2/3 in $A W G N, V=30,60$ and $90 \mathrm{~km} / \mathrm{h}$

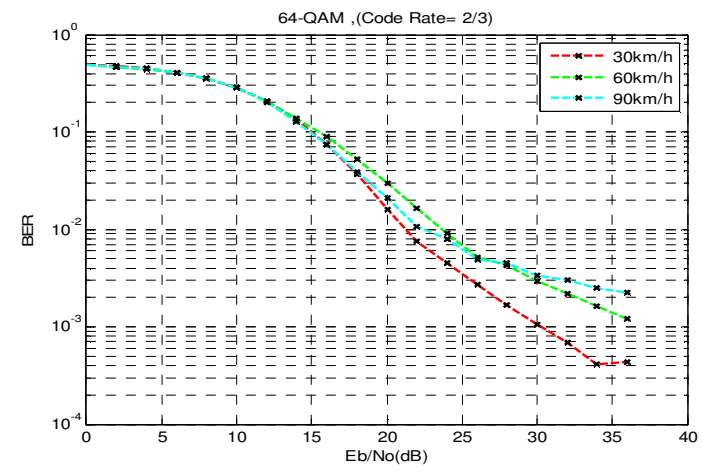

Figure 6.20: BER against $\mathrm{E}_{\mathrm{b}} / \mathrm{N}_{0}$ for 64-QAM-1/2 in Fading channel, $\mathrm{V}=30,60$ and $90 \mathrm{~km} / \mathrm{h}$

\subsection{Capacities of relay and direct links}

This sub-section focused on link capacities in AWGN and multipath fading channels. Figure 6.21, presents link capacities of relay and direct links in pure AWGN non-fading channel while figure 6.22, shows the link capacities in multipath fading channel. From our results obtained, it is observed that relay links offer higher capacity compared to direct links. However, both link capacities reduce marginally in multipath fading channels.

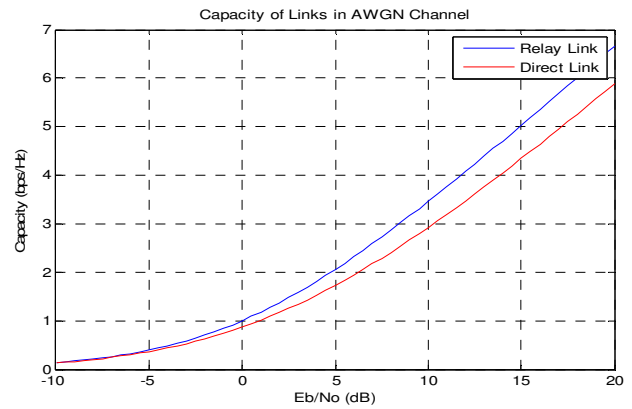

Figure 6.21: Link Capacities of Relay and Direct links against $\mathrm{E}_{\mathrm{b}} / \mathrm{N}_{0}$ in pure $\mathrm{AWGN}$ channel

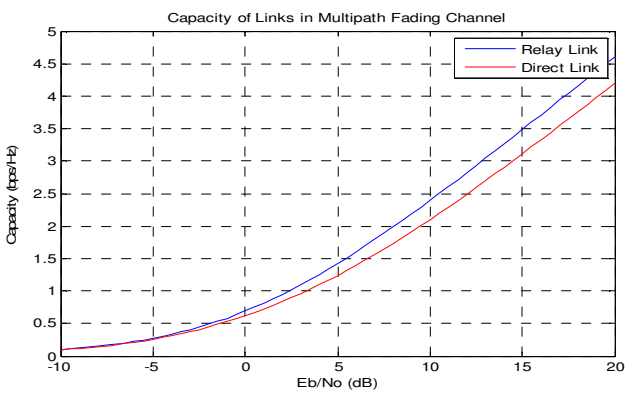

Figure 6.22: Link Capacities of Relay and Direct links against $\mathrm{E}_{\mathrm{b}} / \mathrm{N}_{0}$ in pure Multipath Fading channel

\section{Conclusions}

The results show how various network parameters such as relay deployment, modulation and coding profiles, multipath fading and speed of users affect link performance. We explored two different scenarios with regards to throughput and capacity enhancement; an MS communicating directly with a BS and an MS communicating with a BS through RS.

In terms of link performance, relay link performed appreciably better than direct link in all performance measures studied (i.e. BER, spectral efficiency and link capacity). This is attributed to an increase in bits energy to noise ratio that relays offer which is essential for high data rate transmission. In terms of multipath fading, it was concluded that fading channels significantly had lower performance compared to non-fading AWGN channel. Mobile WiMAX is more affected by the channel because of Doppler shift. To study different speeds, we used speeds of 30, 60 and 90 $\mathrm{km} / \mathrm{h}$ and noted that as the speed increases, the performance of the system decreases. 
Latencies on both links were not considered in this work due to a negligible delay difference in the single cell scenario.

In conclusion, the impact of deploying relays was thoroughly evaluated through link level analysis. The analytical framework was presented highlighting on improved performance on BER, Spectral Efficiency and Capacity as a benefit of using relay enhanced network. The results also show how factors such as speed and fading affect performance. The simulation results will serve as a baseline for future work on coverage and cell edge problems and their countermeasures. Future work may incorporate real world involvement where drive test and optimization tools can be used to achieve a more practical result.

\section{REFERENCES}

[1] P. Ramjee, and F. J. Velez. "OFDMA WiMAX Physical Layer." InWiMAX Networks, pp. 63-135. Springer Netherlands, 2010.

[2] V. Ruby, and P. Garg. "Interpretation of IEEE 802.16 e (Wimax). "GJCST-E: Network, Web \& Security 13, no. 10 (2013).

[3] H.-C. Lu, W. Liao, and F.Y.-S Lin. "Relay Station Placement Strategy in IEEE 802.16j WiMAX Networks," Communications, IEEE Transactions on , vol.59, no.1, pp.151,158, January 2011

[4] K. D. Satish, and N. Nagarajan. "A Survey on Technical Issues in IEEE 802.16 j Mobile Multi-hop Relay Networks." SmartCR 1, no. 1 (2011): 12-33.

[5] IEEE Standard for Local and metropolitan area networks Part 16: Air Interface for Broadband Wireless Access Systems Amendment 1: "Multihop Relay Specification," IEEE Std 802.16j-2009 (Amendment to IEEE Std 802.16-2009), vol., no., pp.1,290, June 122009

[6] J. -Y. Chang. "An accelerating handover process scheme for IEEE $802.16 \mathrm{j}$ multi-hop relay networks." EURASIP Journal on Wireless Communications and Networking 2013, no. 1 (2013): 112 .

[7] V. Genc, S. Murphy, Y. Yu, and J. Murphy. "IEEE 802.16J relay- based wireless access networks : an overview". Wireless Communications, IEEE, Vol.15, 2008.

[8] W. S. Peters, and W. H. Robert "The future of WiMAX : Multihop relaying with IEEE 802.16j". Communications Magazine, IEEE, January 2009.

[9] R. Pabst, B. Walke, D. Schultz, P. Herhold, H. Yanikomeroglu, S. Mukherjee, H. Viswanathan, M. Lott, W. Zirwas, M. Dohler, H. Aghvami, D. Falconer, and G. Fettweis, "Relay-based deployment concepts for wireless and mobile broadband radio," IEEE Communications Magazine, vol. 42, pp. 8089, September 2004.

[10] N. Esseling, B. Walke, and R. Pabst, "Fixed relays for next generation wireless systems," Emerging Location Aware Broadband Wireless Ad Hoc Networks, Springer Science and Business Media, pp. 71-91, 2005.

[11] H. Li, M. Lott, M. Weckerle, W. Zirwas, and E. Schulz, "Multihop communications in future mobile radio networks," in The 13th IEEE International Symposium on Personal, Indoor and Mobile Radio Communications (PIMRC), September 2002.

[12] H. Wei and R. Gitlin, "Two-hop-relay architecture for next-generation WWAN/WLAN integration," IEEE Wireless Communications, vol. 11, pp. 24-30, April 2004.

[13] X. J. Li and P. Chong, "A dynamic channel assignment scheme for TDMA-based multihop cellular networks," IEEE Transactions on Wireless Communications, vol. 7, pp. 1999-2003, June 2008.

[14] J. De Bruyne, W. Joseph, L. Verlook, and L. Martens. "Measurements and evaluation of the network performance of a fixed WiMAX system in a suburban environment". IEEE International Symposium on Wireless Communication Systems (ISWCS), 2008.

[15] K. Haneda, V. M. Kolmonen, T. Riihonen, R. Wichman, P. Vainikainen, and J. I. Takada. "Evaluation of relay transmission in outdoor-to-indoor propagation channels." In European Cooperation in Science and Technology Workshop (COST) (Vol. 2100). 2008

[16] R. Patil. "On throughput performance of decode and forward cooperative relaying with packet combining and ARQ." International Journal of Computer Networks and Communications (IJCNC), 4(3), 57-67. (2012).

[17] K. Park, H. S. Ryu, C. G. Kang, D. Chang, S. Song, J. Ahn, and J. Ihm. "The performance of relayenhanced cellular OFDMA-TDD network for mobile broadband wireless services." EURASIP Journal on Wireless Communications and Networking, 2009. 
[18] R. Hu, C. Hu, J. Jiang, X. Xie, and L. Song. "Full-duplex mode in amplify-and-forward relay channels: outage probability and ergodic capacity." International Journal of Antennas and Propagation, 2014.

[19] P. Gong, P. Xue, D. Park, and D. K. Kim. "Optimum power allocation in a nonorthogonal amplifyand-forward relay-assisted network." Vehicular Technology, IEEE Transactions on, 60(3), 890-900.

[20] A. Azari, J. S. Harsini, and F. Lahouti. "Power Allocation in Multi-hop OFDM Transmission Systems with Amplify-and-Forward Relaying: A Unified Approach.” arXiv preprint arXiv:1508.01216. 2015

[21] A. So and B. Liang, "Enhancing WLAN capacity by strategic placement of Tetherless relay points," IEEE Transactions on Mobile Computing, vol. 6, pp. 522-535, May 2007.

[22] A. So and B. Liang, "Optimal placement of relay infrastructure in heterogeneous wireless mesh networks by Bender's decomposition," in International Conference on Quality of Service in Heterogeneous Wired/Wireless Networks (QShine), 2006.

[23] L. C. Wang, W.-S. Su, J.-H. Huang, A. Chen, and C.-J. Chang, "Optimal relay location in multi-hop cellular systems," IEEE Wireless Communications and Networking Conference (WCNC), April 2008.

[24] E. Amaldi, A. Capone, and F. Malucelli, "Planning UMTS base station location: optimization models with power control and algorithms," IEEE Transactions on Wireless Communications, vol. 2, pp. 939952, September 2003.

[25] K. Cho, W. Lee, D. Yoon, H. S. Jin, K. Kim, and S. K. Park. "Frequency Allocation Scheme for Interference Avoidance in Cellular System with Fixed Relay," Digital Telecommunications, 2008. ICDT '08. The Third International Conference on , vol., no., pp.130,134, June 29 2008-July 52008

[26] D. Niyato, E. Hossain, D. I. Kim, and Z. Han. "Joint Optimization of Placement and Bandwidth Reservation for Relays in IEEE 802.16j Mobile Multihop Networks". IEEE International Conference on Communications (ICC), June 2009.

[27] B. Lin, P. Han Ho, Xie, L. Liang, and X. Shen. "Optimal relay station placement in IEEE 802.16j networks". IEEE International Conference on Wireless Communications and Mobile Computing (IWCMC), 2007.

[28] B. Lin, P. Han Ho, Xie, L. Liang, and X. Shen. "Relay Station Placement in IEEE 802.16j Dual-Relay MMR Networks". IEEE International Conference on Communications (ICC), May 2008.

[29] H. -C. Lu, W. Liao, and F.Y. Lin. "Relay Station Placement Strategy in IEEE 802.16j WiMAX Networks". IEEE Transactionon Communications, Vol.59 :151-158, January 2011.

[30] L. Huang, M. Rong, H. Shi, and D. Yu, "Comparison of two frequency reuse schemes in fixed relay system," in International Conference on Wireless Communications, Networking and Mobile Computing (WiCOM), September 2005.

[31] V. Roman, "Frequency reuse and system deployment in local multipoint distribution service," IEEE Personal Communications, vol. 6, pp. 20-27, December 1999.

[32] H.-M. Zimmermann, J. Eberspacher, and M. Lott, "Frequency reuse for cellular multi-hop networks," in IEEE 62nd Vehicular Technology Conference (VTC), September 2005.

[33] S.-S. Wang, H.-C. Yin, and S.-T. Sheu, "Symmetric path selection in IEEE 802.16 multi-hop relay networks with error prone links," IEEE 4th International Conference on Circuits and Systems for Communications (ICCSC), May 2008.

[34] S. Ann, K. G. Lee, and H. S. Kim. "A Path Selection Method in IEEE 802.16j Mobile Multi-hop Relay Networks". 2008 International Symposium on a World of Wireless, Mobile and Multimedia Networks, 2008.

[35] S. Ann, K. G. Lee, and H. S. Kim, “A path selection method in IEEE 802.16j mobile multi-hop relay networks," 2nd International Conference on Sensor Technologies and Applications (SENSORCOMM), August 2008.

[36] V. Genc, S. Murphy, and J. Murphy. "Performance Analysis of Transparent Relays in 802.16j MMR Networks". Modeling and Optimization in Mobile, Ad Hoc, and Wireless Networks and Workshops. WiOPT 6th International Symposium on, August 2008.

[37] L. Erwu, W. Dongyao, L. Jimin, S. Gang, and J. Shan, "Performance evaluation of bandwidth allocation in 802.16j mobile multi-hop relay networks," IEEE 65th Vehicular Technology Conference (VTC), April 2007.

[38] J. Z. Zhifeng Tao, Koon Hoo Teo, "Aggregation and concatenation in IEEE 802.16j mobile multihop relay (MMR) networks," IEEE Mobile WiMAX Symposium, March 2007.

[39] S.-J. Lin, W. H. Sheen, I.-K. Fu, and C. chi Huang, "Resource scheduling with directional antennas for multi-hop relay networks in manhattan-like environment," IEEE Mobile WiMAX Symposium, March 2007. 
[40] I.-K. Fu, W.-H. Sheen, and F.-C. Ren, "Deployment and radio resource reuse in IEEE 802.16j multihop relay network in manhattan-like environment," 6th International Conference on Information, Communications and Signal Processing (ICICS), December 2007.

[41] Y. Sun, Y. Bian, P. Strauch, and A. Nix, "Study of radio resource sharing for future mobile WiMAX applications with relays," IEEE Mobile WiMAX Symposium, March 2007.

[42] M. Salem, A. Adinoyi, M. Rahman, H. Yanikomeroglu, D. Falconer, Y. D. Kim, E. Kim, and Y.-C. Cheong, "An overview of radio resource management in relay-enhanced OFDMA-based networks," IEEE Communications Surveys and Tutorials, 2009.

[43] Z. Tao, A. Li, K. H. Teo, and J. Zhang. "Frame Structure Design for IEEE 802.16j Mobile Multihop Relay (MMR) Networks". Global Telecommunications Conference, IEEE GLOBECOM, December 2007.

[44] C. Hoymann, M. Dittrich, and S. Boebbels. "Dimensioning Cellular Wimax Part I : Singlehop Networks". European Wireless, Paris, France, April 2007.

[45] C. Hoymann, M. Dittrich, and S. Boebbels. "Dimensioning Cellular Wimax Part II : Multihop Networks". European Wireless, Paris, France, April 2007.

[46] B. Upase and M. Hunukumbure. "Dimensioning and Cost Analysis of Multihop Relay-Enabled WiMAX Networks". Futjisu Scientific and Technical Journal, July 2008.

[47] H. Zeng and C. Zhu, "System-level modeling and performance evaluation of multi-hop 802.16j systems," International Wireless Communications and Mobile Computing Conference (IWCMC), August 2008.

[48] E. Visotsky, J. Bae, R. Peterson, R. Berryl, and M. Honig, "On the uplink capacity of an 802.16j system," IEEE Wireless Communications and Networking Conference (WCNC), April 2008.

[49] N. R. Van, and R. Prasad. "OFDM for wireless multimedia communications." Artech House, Inc., 2000.

[50] Q. Zhang, S. Kassam. "Finite-state Markov model for Rayleigh fading channels," IEEE Trans. Commun. 47(11): 1688-1692. 1999.

[51] V. Erceg, K. V. S. Hari. "Channel models for fixed wireless applications," IEEE 802.16 Broadband Wireless Access Working Group, Technical Report, 2001

[52] T. Zhang, L. Xiao, C. Feng, and L. Cuthbert. "System Level Performance of Multiuser Diversity in Cooperative Relay Based OFDMA Networks,"Advances in Mesh Networks, 2009. MESH 2009. Second International Conference on , vol., no., pp.85,89, 18-23 June 2009

[53] L. Xiao, T. Zhang, Y. Zhu, and L. Cuthbert. "Two-Hop Subchannel Scheduling and Power Allocation for Fairness in OFDMA Relay Networks,"Wireless and Mobile Communications, 2009. ICWMC '09. Fifth International Conference on , vol., no., pp.267,271, 23-29 Aug. 2009 